\title{
Elevated expression of the leptin receptor ob-R may contribute to inflammation in patients with ulcerative colitis
}

\author{
YAN-MING TIAN $^{1 *}$, SI-YU TIAN $^{1 *}$, DONG WANG ${ }^{2}$, FANG CUI $^{1}$, XIANG-JIAN ZHANG ${ }^{3}$ and YI ZHANG $^{1,3}$ \\ ${ }^{1}$ Department of Physiology, Hebei Medical University, Shijiazhuang, Hebei 050017; \\ ${ }^{2}$ Department of Gastroenterology, Second Hospital of Hebei Medical University; ${ }^{3}$ Hebei Collaborative \\ Innovation Center for Cardio-Cerebrovascular Disease, Shijiazhuang, Hebei 050000, P.R. China
}

Received May 25, 2019; Accepted September 10, 2019

DOI: $10.3892 / \mathrm{mmr} .2019 .10720$

\begin{abstract}
The effect of leptin on ulcerative colitis (UC) has been controversial. The present study aimed to investigate the role of leptin and its receptor ob-R in UC and the underlying mechanism of this role. The level of serum leptin and the protein expression of the leptin receptor ob- $\mathrm{R}$ in the colonic mucosa were determined in patients with UC. Experimental colitis was induced through intrarectal administration of 2,4,6-trinitrobenzene sulfonic acid (TNBS) in leptin receptor-deficient Zucker rats (LR-D). The body weight, disease activity index, colon length, and macroscopic and histopathological appearance were evaluated. Furthermore, the myeloperoxidase (MPO) enzyme activity and cytokine levels in colon tissues were also determined. The expression of the signal transducer and activator of transcription 3 (STAT3), phosphorylated STAT3 (p-STAT3), nuclear factor (NF)- $\kappa$ B-p65, and Ras homolog gene family member A (RhoA) proteins in colon tissues was assessed. The results revealed that the expression of the leptin receptor ob-R was increased in the colonic mucosa but the serum leptin level was not altered in patients with UC compared with healthy volunteers. The severity of experimental colitis, represented by body weight loss, disease activity index, colon length, and macroscopic and histological changes, was ameliorated in LR-D rats compared with the wild-type (WT) rats. Moreover, the MPO activity; levels of cytokines including interleukin (IL)-1 $\beta$, IL-6, and tumor necrosis factor- $\alpha$; and expression of p-STAT3, NF- $\kappa$ B, and RhoA proteins were reduced in colon tissues of LR-D rats compared with WT rats. In conclusion, activation of the leptin receptor ob- $\mathrm{R}$ is an important pathogenic mechanism of $\mathrm{UC}$,
\end{abstract}

Correspondence to: Professor Yi Zhang, Department of Physiology, Hebei Medical University, 361 Zhong Shan Dong Road, Shijiazhuang, Hebei 050017, P.R. China

E-mail: zhyhenryphy@163.com

*Contributed equally

Key words: inflammatory bowel disease, ulcerative colitis, leptin, leptin receptor, $\mathrm{NF}-\kappa \mathrm{B}, \mathrm{RhoA}$ and leptin receptor deficiency may provide resistance against TNBS-induced colitis by inhibiting the NF- $\kappa \mathrm{B}$ and RhoA signaling pathways.

\section{Introduction}

Inflammatory bowel disease (IBD), which includes ulcerative colitis (UC) and Crohn's disease (CD), is a common gastrointestinal disease characterized by intestinal inflammation and intestinal mucosal injury (1). Although infection, environmental factors, heredity, and immunological abnormalities have been proposed as possible causes, the pathogenesis of IBD remains unclear (2). Generally, aminosalicylic acid, corticosteroids, immunomodulators, and antibiotics may cause temporary remission of IBD; however, the curative effect of these therapies is not evident (3). Moreover, some adverse effects may arise during treatment. Therefore, it is necessary to investigate the pathogenesis and treatments of IBD.

Leptin is a satiety hormone primarily produced by adipose tissue and exerts many biological effects by binding to its receptor, ob-R. The primary function of leptin is controlling appetite and adiposity (4). Recently, leptin was revealed to be involved in inflammatory responses in diseases such as IBD (5). The effects of leptin on IBD, however, are controversial. For example, Singh et al (6) reported that the level of leptin was increased in a rodent colitis model and that colitis was ameliorated by a leptin antagonist, which suggests that leptin has proinflammatory action. However, some studies have revealed that leptin has anti-inflammatory effects in rats with colitis $(7,8)$. In previous clinical studies, the level of leptin in patients with IBD was reported to be increased (9), decreased (10) or unaltered (11). Therefore, further studies on leptin and IBD in the clinical setting and in experimental animals are required.

The aim of the present study was to investigate the role of leptin and the leptin receptor ob-R, as well as the underlying mechanism of this role, in patients with $\mathrm{UC}$ and in a rat colitis model.

\section{Materials and methods}

Patients and samples. Blood samples and colonic mucosa biopsy specimens were obtained from 11 Chinese Han 
patients with active UC (22-83 years old; female 45.5\%; from the inpatient and outpatient clinics of the Second Hospital of Hebei Medical University) and 19 healthy Chinese Han volunteers (26-67 years old; female 47.4\%) between March 2019 and May 2019. The diagnosis of UC was based on standard clinical, endoscopic, and histological criteria (12). Patients with any other inflammation, metabolic disease, and pregnancy were excluded from the study. The patients did not receive any steroid treatments at least 1 month before enrolment. The healthy control (HC) volunteers had no gastrointestinal discomfort and had normal colonic mucosa under colonoscopy. The procedures and study were approved by the Ethics Committee of the Second Hospital of Hebei Medical University and written informed consent was obtained from all patients.

The level of serum leptin was assessed using ELISA (cat. no. EK197-96; LiankeBio). Furthermore, the protein expression of the leptin receptor ob- $\mathrm{R}$ in the colonic mucosa was determined using the western blot analysis method.

Animals and induction of colitis. Adult male leptin receptor-deficient (LR-D) Zucker rats (weighing 500-600 g; 15 weeks old; purchased from Charles River Laboratories) and age-matched wild-type (WT) control Zucker rats (weighing $300-400 \mathrm{~g}$ ) were used in the present study. The animals were housed in a temperature-controlled room $\left(22 \pm 1^{\circ} \mathrm{C}\right)$ with a 12-h light/dark cycle and had free access to water and food. All experiments were conducted in accordance with the Guide for the Care and Use of Laboratory Animals (National Research Council, 1996), and all techniques and procedures were reviewed and approved by the Hebei Medical University Institutional Animal Care and Use Committee.

Experimental colitis was induced in a rat model according to a previously described procedure (13). Briefly, after a 24-h fast, the rats were anesthetized with isoflurane and a medical-grade polyurethane cannula (external diameter, $2 \mathrm{~mm}$ ) was inserted into the anus and advanced to $8 \mathrm{~cm}$ proximal to the anal verge. 2,4,6-Trinitrobenzene sulfonic acid (TNBS; Sigma-Aldrich; Merck $\mathrm{KGaA}$ ), dissolved in $50 \%$ ethanol, was instilled into the colon $(100 \mathrm{mg} / \mathrm{kg})$ to induce colitis. At the end of instillation, the animals were maintained in a head-down position for 2 min to prevent leakage of the instilled TNBS. The body weight was measured daily. After the experiments, all rats were euthanized using an overdose of sodium pentobarbital (66 mg/kg, intraperitoneal).

Assessment of colitis. The disease activity index (DAI) was determined based on stool consistency $(0=$ firm, $1=$ loose, $2=$ diarrhea $)$ and occult blood $(0=$ no blood, $1=$ occult blood, $2=$ gross rectal bleeding) (14). The full colon was removed and the length was measured. After being cut longitudinally, colon tissues were rinsed in physiological saline to remove fecal residue. The gross appearance of colitis was evaluated based on adhesions (score 0-2), ulcer, and inflammation (score 0-8) (15).

Colon histology. Sections of inflamed colon were collected and immersed in $4 \%$ paraformaldehyde at $4^{\circ} \mathrm{C}$ for $48 \mathrm{~h}$ and then dehydrated in gradient ethanol step by step. After embedding in wax, tissues were sectioned at 6- $\mu \mathrm{m}$ thickness
Table I. Clinical characteristics.

\begin{tabular}{lccc}
\hline Characteristic & $\begin{array}{c}\text { Ulcerative } \\
\text { colitis }(\mathrm{n}=11)\end{array}$ & $\begin{array}{c}\text { Healthy } \\
\text { control }(\mathrm{n}=19)\end{array}$ & P-value \\
\hline Female, \% & 45.5 & 47.4 & 0.919 \\
Age, years & $43.5 \pm 5.0$ & $44.6 \pm 2.8$ & 0.847 \\
BMI, kg/m & $20.6 \pm 1.3$ & $20.9 \pm 0.7$ & 0.778 \\
\hline
\end{tabular}

using a microtome and then stained with hematoxylin-eosin at room temperature for $10 \mathrm{~min}$. Pathological changes in the colon were imaged with a light microscope digital camera (magnification, x50). Microscopic scores were evaluated based on morphological features according to the severity of inflammation (score 0-3), extent of inflammation (0-3), and crypt damage (0-4) (16).

Colon cytokine and myeloperoxidase (MPO) analysis. Sections of inflamed colon were homogenized with saline, and the supernatant was obtained after centrifugation for $15 \mathrm{~min}$ at $1,004 \mathrm{x} \mathrm{g}$ at $4^{\circ} \mathrm{C}$. The interleukin-1 $\beta$ (IL-1 $\beta$; cat. no. EK201B/3-96), IL-6 (cat. no. EK306/3-96) and tumor necrosis factor- $\alpha$ (TNF- $\alpha$; cat. no. EK382/3-96) levels, and the MPO (cat. no. EK2133/2-96) activity were detected using commercial ELISA kits (LiankeBio).

Western blot analysis. The collected colon tissues were homogenized in a lysis buffer (Beyotime Institute of Biotechnology), and the protein level was determined using the Bradford assay. Protein samples $(50 \mu \mathrm{g})$ were separated by SDS-PAGE on $10 \%$ gels, transferred to a polyvinylidene fluoride membrane that was blocked for $1 \mathrm{~h}$ with $5 \%(\mathrm{w} / \mathrm{v})$ non-fat milk in TBS, and incubated with primary antibodies against the leptin receptor ob-R (1:50; cat. no. sc-8391; Santa Cruz Biotechnology, Inc.), signal transducer and activator of transcription 3 (STAT3; 1:1,000; cat. no. 9139; Cell Signaling Technology, Inc.), phosphorylated STAT3 (pSTAT3; 1:2,000; cat. no. 9145; Cell Signaling Technology, Inc.), nuclear factor (NF)-кB-p65 (1:2,000; cat. no. ab16502; Abcam) and Ras homolog gene family member A (RhoA; 1:2,000; cat. no. ab219371; Abcam) overnight at $4^{\circ} \mathrm{C}$. The same membrane was stripped and re-blotted with an anti- $\beta$-actin antibody (1:5,000; cat. no. T0022; Affinity Biosciences) for normalization. The membranes were then incubated with corresponding secondary antibodies (1:5,000, Goat Anti-Rabbit, cat. no. ab6721; 1:5,000, Rabbit Anti-Mouse, cat. no. ab6728; both Abcam) for $1 \mathrm{~h}$ at room temperature. Blots were developed using the chemiluminescent detection method (Immobilon Western HRP; EMD Millipore). The protein blots were quantified by densitometry using ImageJ (version 1.46; National Institutes of Health) and normalized to $\beta$-actin.

Statistical analysis. All statistical analyses were performed with GraphPad Prism, version 6 (GraphPad Software, Inc.). The sex ratio with respect to clinical characteristics was assessed using the $\chi^{2}$ test. Differences in measurement data were assessed using one-way analysis of variance followed by 

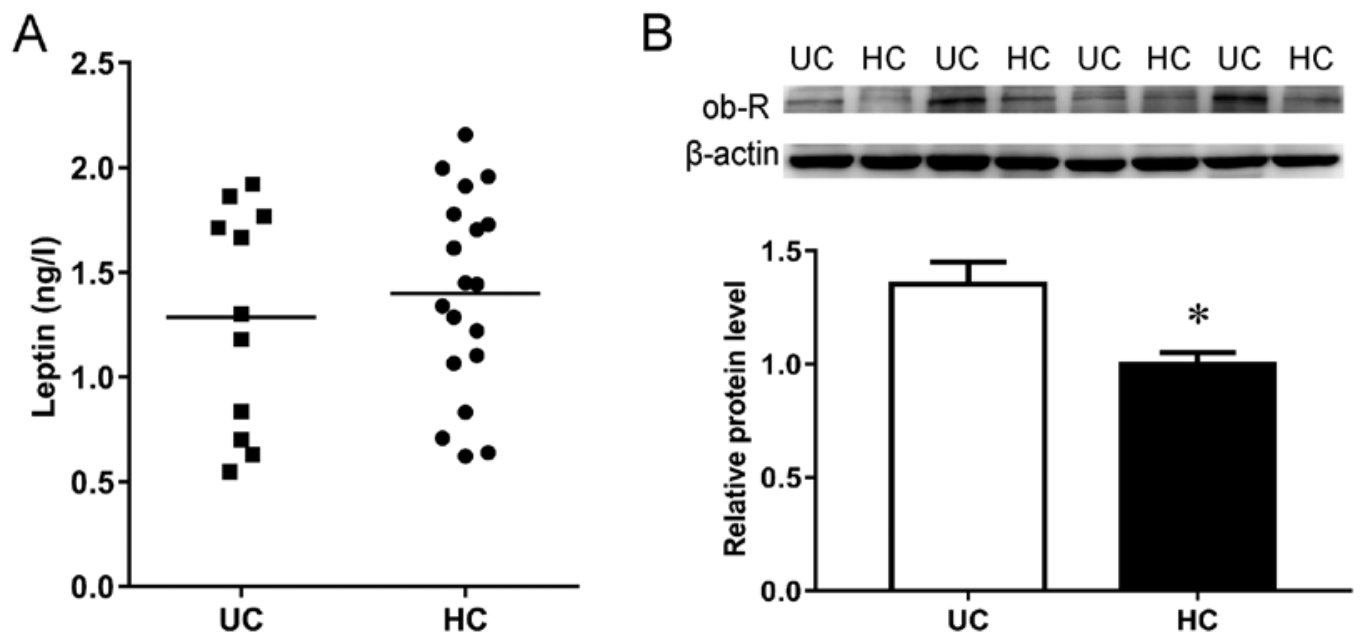

Figure 1. Determination of leptin signaling in patients with UC. (A) Serum leptin levels in patients with UC ( $\mathrm{n}=11)$ and in healthy control volunteers ( $\mathrm{n}=19)$. (B) Leptin receptor ob-R protein expression in the colonic mucosa of patients with $\mathrm{UC}(\mathrm{n}=4)$ and healthy control volunteers $(\mathrm{n}=4)$. ${ }^{*} \mathrm{P}<0.05$ vs. patients with UC. UC, ulcerative colitis.

A
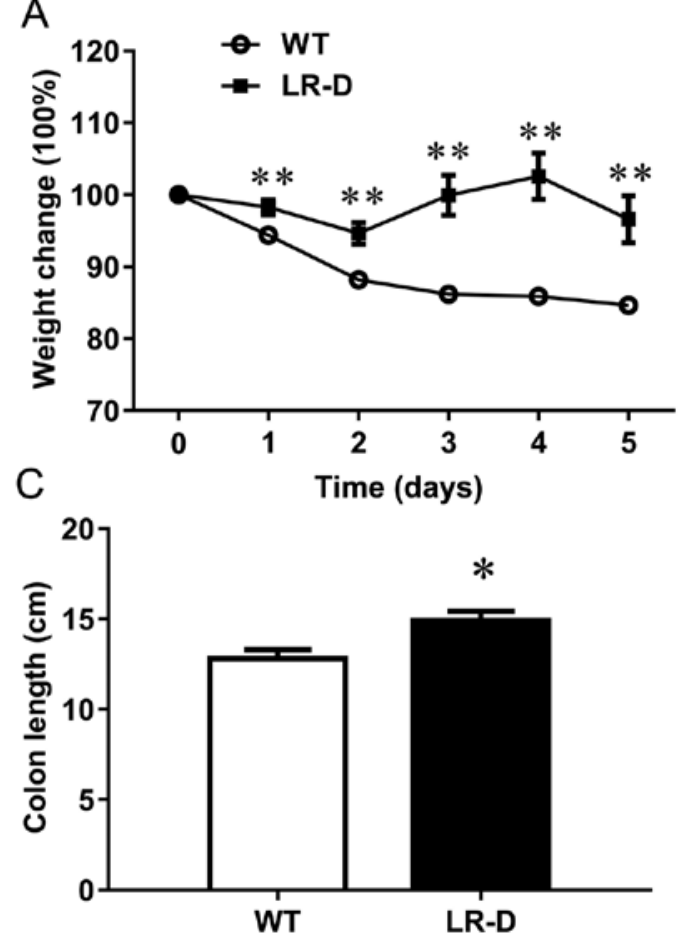

B

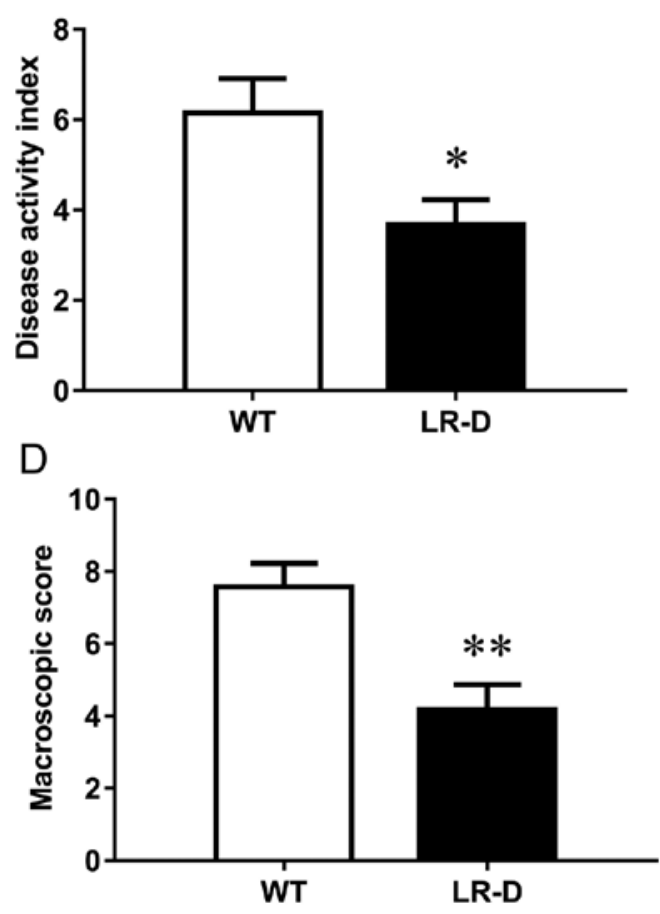

Figure 2. General performance of animals after treatment with TNBS. (A) TNBS induced body weight changes over 5 days. (B) Disease activity index in the colon after TNBS treatment. (C) Colon length. (D) Gross appearance of the colon after TNBS treatment; $n=6$ in each group. ${ }^{*} \mathrm{P}<0.05,{ }^{* *} \mathrm{P}<0.01$ vs. the $\mathrm{WT}$ group. TNBS, 2,4,6-trinitrobenzene sulfonic acid; WT, wild-type.

Tukey's test or two-tailed Student's t-test. Data are presented as the mean \pm standard error of mean, unless indicated otherwise. $\mathrm{P}<0.05$ was considered to indicate a statistically significant difference.

\section{Results}

Leptin receptor is elevated in patients with UC. There were no significant differences in age, sex, body mass index (BMI), and serum leptin level between patients with UC and the HC group (Table I; Fig. 1A). However, the expression of the leptin receptor $\mathrm{ob}-\mathrm{R}$ in the colonic mucosa was increased in patients with UC compared with the HC group (Fig. 1B), indicating that leptin signaling was enhanced in patients with UC.

Experimental colitis was ameliorated in LR-D rats. After the instillation of TNBS in the colon, all rats exhibited decreased body weight; however, the weight decrease was much more severe in WT rats than in LR-D rats (Fig. 2A). In addition, LR-D rats exhibited low DAI scores, long colon lengths, and low scores in the gross appearance of the colon compared with 


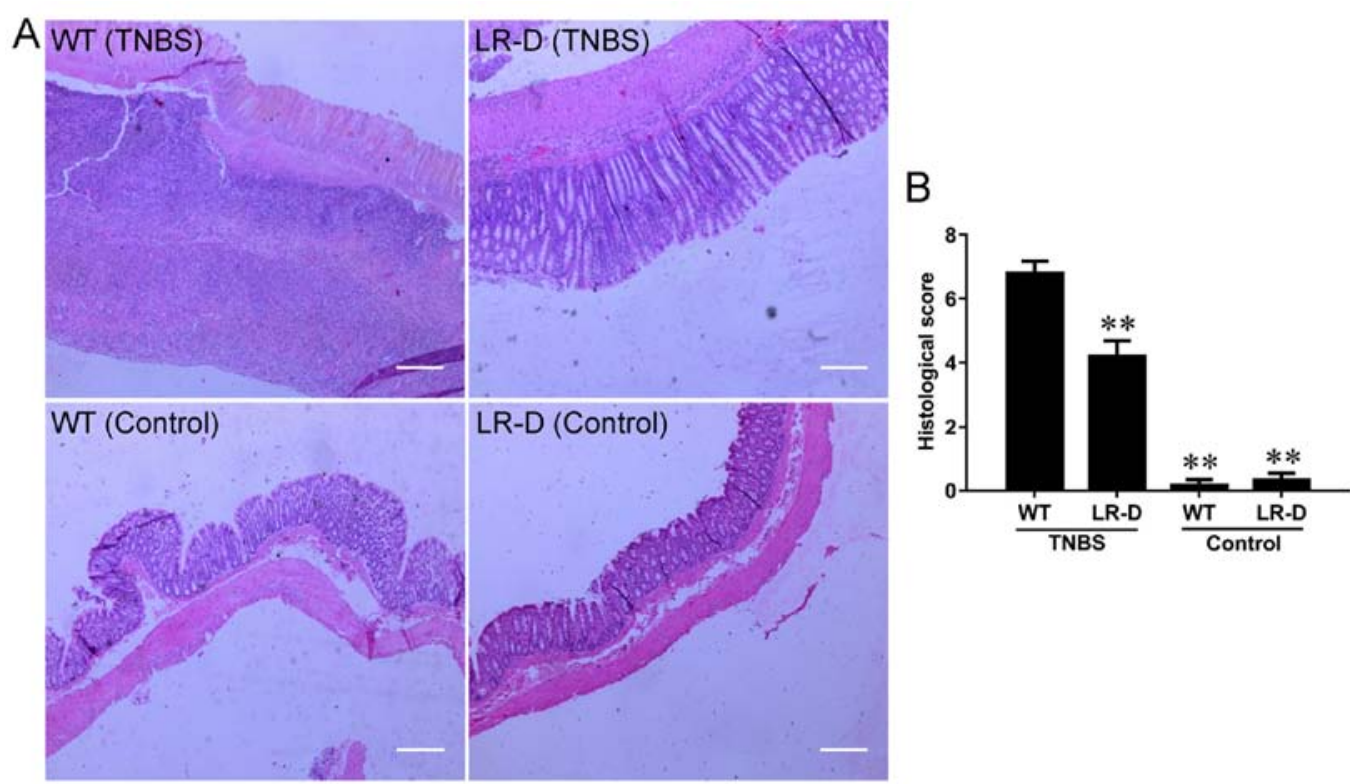

Figure 3. Histological analysis of colon tissues. (A) Representative hematoxylin-eosin-stained colon sections before and 5 days after treatment with TNBS Scale bar, $400 \mu \mathrm{m}$. (B) Histological scores of colon tissues before and after TNBS treatment; $\mathrm{n}=6$ in each group. ${ }^{* *} \mathrm{P}<0.01 \mathrm{vs.} \mathrm{the} \mathrm{WT} \mathrm{group.} \mathrm{TNBS,}$ 2,4,6-trinitrobenzene sulfonic acid; WT, wild-type.

the WT control rats (Fig. 2). These results indicated that the general condition was improved in LR-D rats than in WT rats after TNBS instillation.

Colonic histological appearance is improved in LR-D rats. Histological analysis revealed an intact colonic mucosal epithelium and neatly arranged glands before TNBS instillation in both LR-D and WT rats (Fig. 3A). After TNBS treatment, the rats exhibited typical colitis features, such as increased inflammation in the mucosa, thickening and edema with loss of crypts in the submucosa, and inflammatory infiltration in the muscularis (Fig. 3A). However, the microscopic inflammation scores were higher in WT rats than in LR-D rats (Fig. 3B), indicating that LR-D rats had improved histological outcomes than WT rats.

Inflammation is reduced in LR-D rats. The expression of inflammatory cytokines including IL- $1 \beta$, TNF- $\alpha$, and IL-6 was significantly lower in LR-D rats than in WT rats (Fig. 4A-C). The reduced inflammation in LR-D rats was also confirmed by the lower activity of MPO, a marker of neutrophil infiltration, in the colon of LR-D rats than that in WT rats (Fig. 4D). This indicated that leptin receptor deficiency could reduce the level of inflammation in experimental colitis.

Protein expression in colon tissue. pSTAT3/STAT3 signaling has been suggested to mediate the major effects of ob-R. Western blot analysis was used to assess the expression of STAT3 protein in the two groups. pSTAT3 (Fig. 5A) was significantly downregulated in the colon tissues of LR-D rats compared with that in WT rats, which reliably suggested the correlation of pSTAT3 with ob-R deficiency.

The expression of NF- $\kappa \mathrm{B}-\mathrm{p} 65$, a member of the NF- $\kappa \mathrm{B}$ transcription factor family that is crucial in inflammatory diseases, was downregulated in TNBS-induced colitis tissues of LR-D rats compared with that in WT rats (Fig. 5B). Furthermore, the expression of RhoA protein was downregulated in colon tissues of LR-D rats compared with that in WT rats (Fig. 5B).

\section{Discussion}

In the present study, leptin signaling was examined in patients with UC. The results revealed that the expression of the leptin receptor ob-R was increased in the colonic mucosa of patients with UC compared with that in healthy volunteers, whereas no difference was revealed in the serum leptin level between the two groups. To clarify the role of the leptin receptor in UC (a type of IBD), an experimental colitis model was developed in LR-D Zucker rats. The results indicated that LR-D rats exhibited a resistance to TNBS-induced colitis compared with WT rats.

Recent studies have revealed that leptin is a pro-inflammatory factor $(5,17)$. However, the serum leptin level in patients with IBD was not consistently reported in clinical studies [increased (9), decreased (10), or unaltered (11)]. The reason for this discrepancy is still unclear. It was reported that inflammatory cytokines stimulate the elevation of the level of leptin with anorexia and body weight loss, which are common findings due to inflammation in patients with IBD (18). There is a close relationship among body weight, plasma leptin and inflammatory cytokines. For example, body weight loss is often associated with a decrease in plasma leptin (19) and inflammatory cytokines enhance the leptin expression. We speculate that the interaction among the three factors determines the level of plasma leptin in different stages of the disease. Thus, the leptin level in the circulatory system cannot reflect the local inflammation status.

Leptin exerts its biological effects by binding to its receptor, ob-R. Unfortunately, most previous studies on leptin signaling focused on leptin and few studies paid attention to ob-R. In the present study, although no difference in the level of serum leptin was revealed between patients with IBD and the HC group, this does not exclude the possibility that 

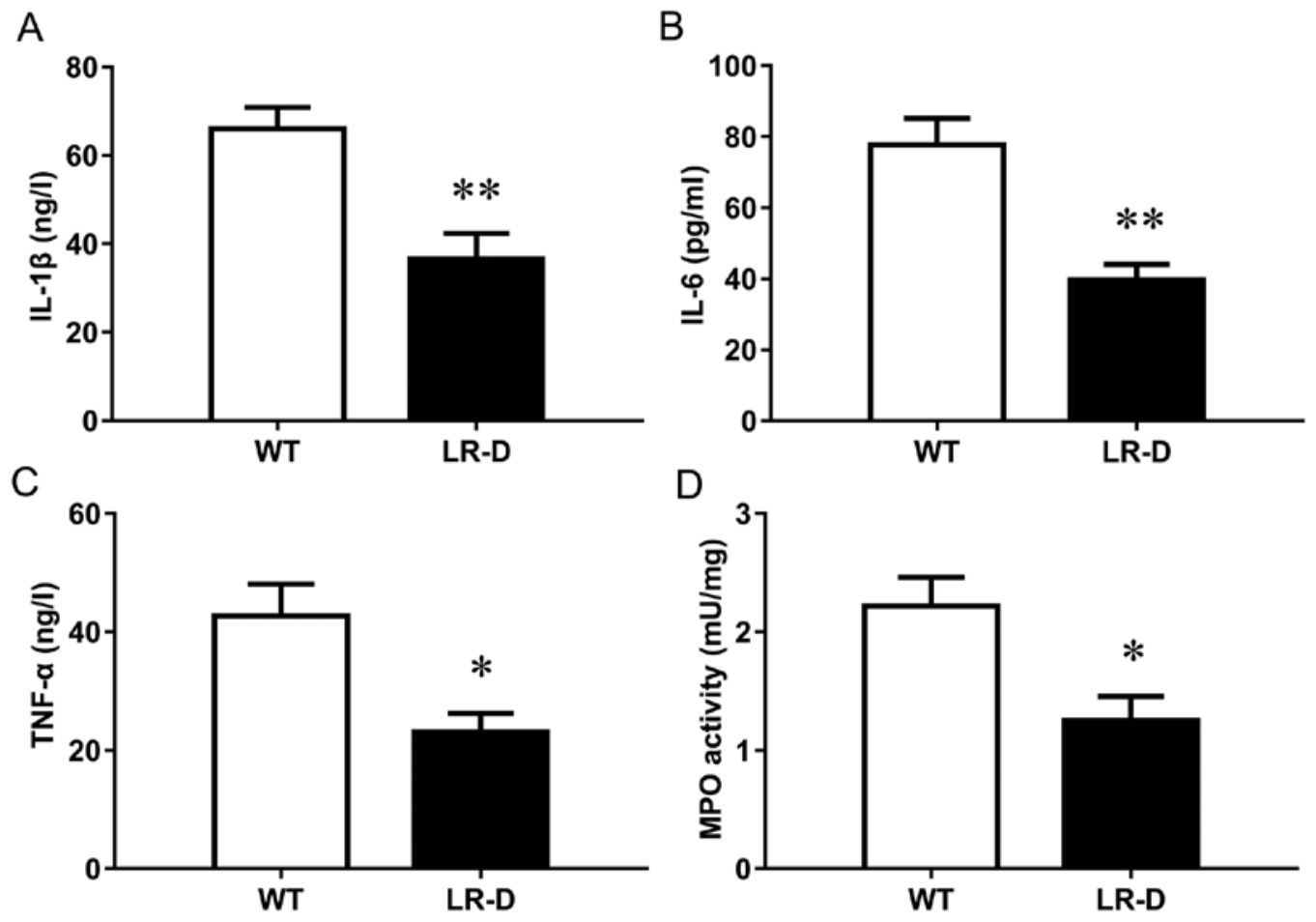

Figure 4. Inflammatory markers in colon tissues after treatment with TNBS. The levels of (A) IL-1 $\beta$, (B) IL-6 and (C) TNF- $\alpha$, and the (D) MPO activity are presented. $\mathrm{n}=6$ in each group. ${ }^{*} \mathrm{P}<0.05,{ }^{* *} \mathrm{P}<0.01$ vs. the WT group. TNBS, 2,4,6-trinitrobenzene sulfonic acid; IL, interleukin; TNF, tumor necrosis factor; MPO, myeloperoxidase; WT, wild-type.

A
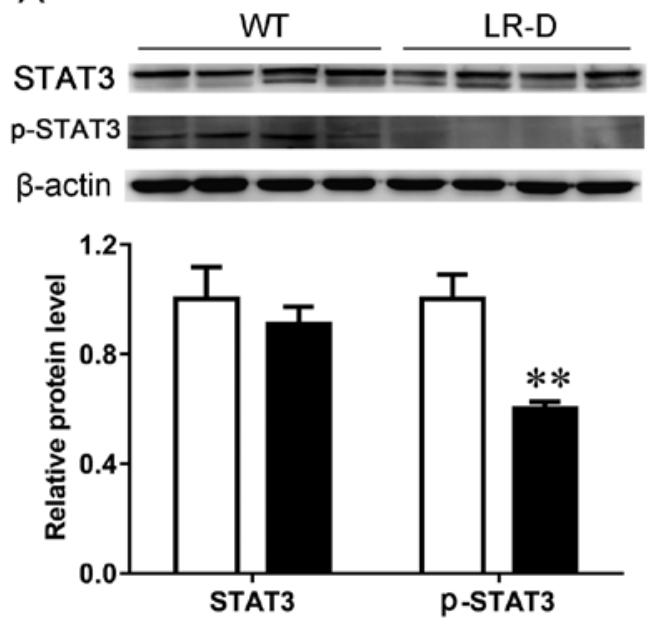

B
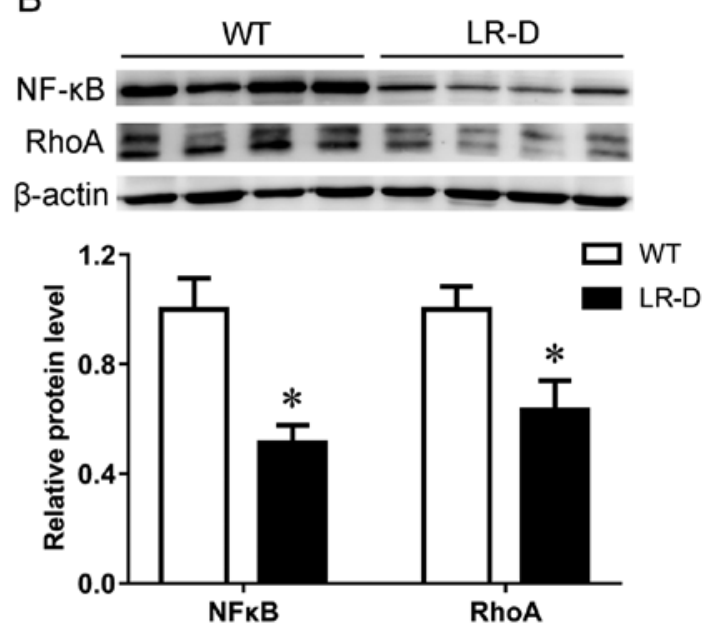

Figure 5. Western blot analysis of the expression of (A) STAT, p-STAT and (B) NF- $\kappa \mathrm{B}$, and RhoA protein in colon tissues; $\mathrm{n}=4$ in each group. ${ }^{*} \mathrm{P}<0.05,{ }^{* *} \mathrm{P}<0.01$ vs. the WT group. STAT, signal transducer and activator of transcription; p-STAT, phosphorylated STAT; NF- $\kappa$ B, nuclear factor- $\kappa$ B; RhoA, Ras homolog gene family member A; WT, wild-type.

the leptin signaling pathway is altered in IBD. Therefore, the expression of the leptin receptor ob- $\mathrm{R}$ in the local inflammatory colonic mucosa of patients with UC was assessed. On the basis of the clinical result that the expression of the leptin receptor ob-R was increased in the colonic mucosa of patients with UC, it was speculated that the leptin signaling pathway is upregulated in IBD. To further confirm whether the leptin receptor contributed to the pathogenesis of IBD, an experimental animal study was performed. An experimental colitis model in LR-D Zucker rats was developed, which is a good model of ob-R deficiency and manifests relatively early-onset obesity (20). The results indicated that LR-D rats were resistant to TNBS-induced colitis compared with WT rats. It was speculated that the resistance to colitis of LR-D rats was due to the leptin receptor deficiency itself rather than obesity, because obesity exacerbates colitis (21). Therefore, the role of the leptin receptor in the pathogenesis of IBD was confirmed in both human and animal models.

Furthermore, the mechanism underlying the alleviation of colitis in LR-D rats was investigated. NF- $\kappa \mathrm{B}$ is a potent pro-inflammatory nuclear transcription factor and a central mediator of immune and inflammatory responses (22). It is well 
known that NF- $\kappa \mathrm{B}$ can upregulate major inflammatory factors and can induce cellular and DNA damage (23). For example, $\mathrm{NF}-\kappa \mathrm{B}$ activity is increased in inflamed intestinal mucosa. Moreover, cytokines that are implicated in IBD, such as TNF- $\alpha$ and IL-1 $\beta$, are potent activators of $N F-\kappa B(24)$. Additionally, many therapies for IBD act, at least in part, through the inhibition of NF- $\kappa \mathrm{B}(25,26)$. Researchers have demonstrated that RhoA and its downstream effector Rho-associated kinase (ROCK) are activated in CD and in TNBS-induced colitis, and that blockade of ROCK can inhibit proinflammatory cytokine production (27). RhoA-dependent signaling plays an important role in inflammatory diseases. It was reported that the blockade of Rho kinase prevents inflammation through NF- $\mathrm{B}$ inhibition in experimental colitis (28). In the present study, consistent with the change in histological and inflammatory factors, $\mathrm{NF}-\kappa \mathrm{B}$ and RhoA were both decreased in inflamed colon tissues of LR-D rats, which indicated that the amelioration of inflammation in LR-D rats with UC may be accomplished by inhibiting the NF- $\kappa \mathrm{B}$ and RhoA signaling pathways. Further studies are required to clarify the relationship between the leptin receptor and NF- $\mathrm{NB}$ and RhoA signaling.

There are also several unresolved issues in the present study. Leptin exerts its biological actions by binding to its long-form receptor ob-R and transmits extracellular signals through the Janus kinase and STAT signaling pathway. However, it is unclear which pathway has a role in the pathogenesis of colitis. Furthermore, the causal relationship between increased expression of leptin receptor ob-R and colonic inflammation was not demonstrated.

In summary, the results of the present study indicate that the activation of the leptin receptor ob- $\mathrm{R}$ is important in the pathogenesis of IBD, and leptin receptor deficiency may provide resistance against TNBS-induced colitis by inhibiting the NF- $\kappa \mathrm{B}$ and RhoA signaling pathways.

\section{Acknowledgements}

Not applicable.

\section{Funding}

The present study was supported by the National Natural Science Foundation of China (grant no. 31671184).

\section{Availability of data and materials}

The datasets used and/or analyzed during the current study are available from the corresponding author on reasonable request.

\section{Authors' contributions}

YMT performed the experiments and drafted the manuscript. SYT performed the experiments and drafted the figures. DW interpreted the results of the experiments. FC performed part of the experiments. XJZ analyzed the data. YZ was responsible for the conception and design of the research, and revised and approved the final manuscript. All authors read and approved the manuscript and agree to be accountable for all aspects of the research in ensuring that the accuracy or integrity of any part of the work are appropriately investigated and resolved.

\section{Ethics approval and consent to participate}

The procedures and study were approved by the Ethics Committee of the Second Hospital of Hebei Medical University and written informed consent was obtained from all patients. All animal experiments were conducted in compliance with the Guide for the Care and Use of Laboratory Animals (National Research Council, 1996), and all techniques and procedures were reviewed and approved by the Hebei Medical University Institutional Animal Care and Use Committee.

\section{Patient consent for publication}

Not applicable.

\section{Competing interests}

The authors declare that they have no competing interests.

\section{References}

1. Ng SC, Shi HY,Hamidi N, Underwood FE, Tang W, Benchimol EI, Panaccione R, Ghosh S, Wu JCY, Chan FKL, et al: Worldwide incidence and prevalence of inflammatory bowel disease in the 21st century: A systematic review of population-based studies. Lancet 390: 2769-2778, 2018

2. Zhou M, He J, Shen Y, Zhang C, Wang J and Chen Y: New frontiers in genetics, gut microbiota and immunity: A Rosetta stone for the pathogenesis of inflammatory bowel disease. Biomed Res Int 2017: 8201672, 2017.

3. Coskun M, Vermeire $\mathrm{S}$ and Nielsen $\mathrm{OH}$ : Novel targeted therapies for inflammatory bowel disease. Trends Pharmacol Sci 38: 127-142, 2017.

4. Münzberg H and Morrison CD: Structure, production and signaling of leptin. Metabolism 64: 13-23, 2015.

5. Abella V, Scotece M, Conde J, Pino J, Gonzalez-Gay MA, Gómez-Reino JJ, Mera A, Lago F, Gómez R and Gualillo O: Leptin in the interplay of inflammation, metabolism and immune system disorders. Nat Rev Rheumatol 13: 100-109, 2017.

6. Singh UP, Singh NP, Guan H, Busbee B, Price RL, Taub DD, Mishra MK, Fayad R, Nagarkatti M and Nagarkatti PS: Leptin antagonist ameliorates chronic colitis in IL-10\% mice. Immunobiology 218: 1439-1451, 2013.

7. Cakir B, Bozkurt A, Ercan F and Yeğen BC: The anti-inflammatory effect of leptin on experimental colitis: Involvement of endogenous glucocorticoids. Peptides 25: 95-104, 2004.

8. Bozkurt A, Cakir B, Ercan F and Yeğen BC: Anti-inflammatory effects of leptin and cholecystokinin on acetic acid-induced colitis in rats: Role of capsaicin-sensitive vagal afferent fibers. Regul Pept 116: 109-118, 2003.

9. Kahraman R, Calhan T, Sahin A, Ozdil K, Caliskan Z, Bireller ES and Cakmakoglu B: Are adipocytokines inflammatory or metabolic mediators in patients with inflammatory bowel disease? Ther Clin Risk Manag 13: 1295-1301, 2017.

10. Trejo-VazquezF,Garza-VelozI, Villela-RamirezGA,Ortiz-CastroY, Mauricio-Saucedo P, Cardenas-Vargas E, Diaz-Baez M, Cid-Baez MA, Castañeda-Miranda R, Ortiz-Rodriguez JM, et al: Positive association between leptin serum levels and disease activity on endoscopy in inflammatory bowel disease: A case-control study. Exp Ther Med 15: 3336-3344, 2018.

11. Ghomraoui FA, Alotaibi ST, Alharthi MA, Asiri SS, Almadi MA, Alharbi OR, Azzam NA, Aljebreen AM, Saeed M, Hajkhder B, et al: Plasma ghrelin and leptin in patients with inflammatory bowel disease and its association with nutritional status. Saudi J Gastroenterol 23: 199-205, 2017.

12. Nikolaus S and Schreiber S: Diagnostics of inflammatory bowel disease. Gastroenterology 133: 1670-1689, 2007.

13. Lin H, Honglang L, Weifeng L, Junmin C, Jiantao Y and Junjing G: The mechanism of alopolysaccharide protecting ulceralive colitis. Biomed Pharmacother 88: 145-150, 2017.

14. Wang J, Chen H, Wang Y, Cai X, Zou M, Xu T, Wang M, Wang J and Xu D: Therapeutic efficacy of a mutant of keratinocyte growth factor-2 on trinitrobenzene sulfonic acid-induced rat model of Crohn's disease. Am J Transl Res 8: 530-543, 2016. 
15. Zhao HM, Wang Y, Huang XY, Huang MF, Xu R, Yue HY, Zhou BG, Huang HY, Sun QM and Liu DY: Astragalus polysaccharide attenuates rat experimental colitis by inducing regulatory $\mathrm{T}$ cells in intestinal Peyer's patches. World J Gastroenterol 22: 3175-3185, 2016.

16. Lu Y, Lin H, Zhang J, Wei J, Sun J and Han L: Sijunzi Decoction attenuates 2, 4, 6-trinitrobenzene sulfonic acid (TNBS)-induced colitis in rats and ameliorates TNBS-induced claudin-2 damage via $\mathrm{NF}-\kappa \mathrm{B}$ pathway in $\mathrm{Caco} 2$ cells. BMC Complement Altern Med 17: 35, 2017.

17. Flatow EA, Komegae EN, Fonseca MT, Brito CF, Musteata FM, Antunes-Rodrigues J and Steiner AA: Elucidating the role of leptin in systemic inflammation: A study targeting physiological leptin levels in rats and their macrophages. Am J Physiol Regul Integr Comp Physiol 313: R572-R82, 2017.

18. Mowat C, Cole A, Windsor A, Ahmad T, Arnott I, Driscoll R, Mitton S, Orchard T, Rutter M, Younge L, et al: Guidelines for the management of inflammatory bowel disease in adults. Gut 60: 571-607, 2011.

19. Pan WW and Myers MG Jr: Leptin and the maintenance of elevated body weight. Nat Rev Neurosci 19: 95-105, 2018

20. Wang B, Chandrasekera PC and Pippin JJ: Leptin- and leptin receptor-deficient rodent models: Relevance for human type 2 diabetes. Curr Diabetes Rev 10: 131-145, 2014.

21. Paik J, Fierce Y, Treuting PM, Brabb T and Maggio-Price L: High-fat diet-induced obesity exacerbates inflammatory bowel disease in genetically susceptible Mdr1a-/- male mice. J Nutr 143: $1240-1247,2013$
22. Sun SC: The non-canonical NF- $\kappa \mathrm{B}$ pathway in immunity and inflammation. Nat Rev Immunol 17: 545-58, 2017.

23. Fan Y, Mao R and Yang J: NF- $\mathrm{BB}$ and STAT3 signaling pathways collaboratively link inflammation to cancer. Protein Cell 4: 176-185, 2013.

24. Hayden MS and Ghosh S: Regulation of NF- $\kappa \mathrm{B}$ by TNF family cytokines. Semin Immunol 26: 253-266, 2014.

25. El-Salhy M and Umezawa K: Effects of AP-1 and NF- $\kappa$ B inhibitors on colonic endocrine cells in rats with TNBS-induced colitis. Mol Med Rep 14: 1515-1522, 2016.

26. Lee C, Kim BG, Kim JH, Chun J, Im JP and Kim JS: Sodium butyrate inhibits the NF-kappa B signaling pathway and histone deacetylation, and attenuates experimental colitis in an IL-10 independent manner. Int Immunopharmacol 51: 47-56, 2017.

27. Segain JP, Raingeard de la Blétière D, Sauzeau V, Bourreille A, Hilaret G, Cario-Toumaniantz C, Pacaud P, Galmiche JP and Loirand G: Rho kinase blockade prevents inflammation via nuclear factor kappa B inhibition: Evidence in Crohn's disease and experimental colitis. Gastroenterology 124: 1180-1187, 2003.

28. Zou Y, Ma L, Zhao Y, Zhang S, Zhou C and Cai Y: Inhibition of Rho kinase protects against colitis in mice by attenuating intestinal epithelial barrier dysfunction via MLC and the NF- $\mathrm{KB}$ pathway. Int J Mol Med 41: 430-438, 2018. 